\title{
Body Image Dissatisfaction and Disordered Eating in Black and White Women
}

\author{
Marisol Perez and Thomas E. Joiner, Jr.* \\ Department of Psychology, Florida State University, Tallahassee, Florida \\ Accepted 9 May 2002
}

\begin{abstract}
Objective: This study predicted and found that body image dissatisfaction and bulimic symptoms have a curvilinear relationship among undergraduate women. Results: For the women in this sample, regardless of race, body image dissatisfaction correlated with bulimic symptoms, such that women who perceived themselves as bigger or smaller than the ideal body size for their ethnic group endorsed bulimic symptoms. Black and white women differed regarding their ethnic group's ideal body image and their self-perceptions of how they compared with the ideal image. Black women tended to report being underweight, whereas white women tended to report being overweight. Discussion: The findings in this study suggest that some black women are not buffered against eating disorders as suggested in previous research. (c) 2003 by Wiley Periodicals, Inc. Int J Eat Disord 33: 342-350, 2003.
\end{abstract}

Key words: body image; eating; ethnicity; bulimia

\section{INTRODUCTION}

According to Usmiani and Daniluk (1997), the discrepancy between the actual and ideal body image is a function of the "culturally defined images of desirable bodily appearances." Following this definition, an individual's self-concept regarding body image is influenced by the degree to which cultural standards are met. Within the United States, research suggests that the cultural standards for the female body ideal are thin (Butler \& Ryckman, 1993; Lamb, Jackson, Cassiday, \& Priest, 1993; Myers \& Biocca, 1992) and steadily thinning (Stice \& Shaw, 1994). Given this, one would expect that women who internalize the cultural standards will have higher rates of eating disorder symptoms, and those who do not internalize the cultural standards will have lower rates of eating disorder symptoms. This is evident in research comparing white and black women within the United States.

Eating disorders are most common in young white women, whereas they are less common in black women (Abrams, Allen, \& Gray, 1993; Akan \& Grilo, 1995; Rucker \& Cash, 1992; Molloy \& Herzberger, 1998; Parker et al., 1995). Research suggests that

${ }^{*}$ Correspondence to: Thomas E. Joiner, Jr., Department of Psychology, Florida State University, Tallahassee, FL 32306-1210.

Published online in Wiley InterScience (www.interscience.wiley.com). DOI: 10.1002/eat.10148

(C) 2003 by Wiley Periodicals, Inc. 
cultural loyalty and strong ethnic identity protects black women from the thin ideal standards of American culture (Parker et al., 1995; Molloy \& Herzberger, 1998). In fact, research shows that black women are less likely to diet (Akan \& Grilo, 1995; Allan, Mayo, \& Michel, 1993; Casper \& Offer, 1990), less fearful of weight gain, and have less negative perceptions on overeating than white women (Casper \& Offer, 1990).

Black women may be protected from the thin ideal standards of American culture, but are they protected from the pressure of attaining their ethnic group's ideal body image? One might expect that black women who are significantly overweight from their ideal body image might display similar dieting, bingeing, and purging symptoms as white women. Given that the black culture has a larger ideal body image than white women, one might also expect that black women who are significantly underweight might feel pressure to meet the ideal body standard and engage in bingeing and disordered eating behaviors, except for purging. Underweight black women are not expected to engage in purging behaviors, because this would obstruct the goal of gaining weight.

Higgins' (1987) theory of the self is consistent with the view that self-relevant discrepancies may affect behavioral outcomes. According to Higgins, the concept of the self consists of many beliefs that can be divided into the actual self, the ideal self, and other forms of the self. Any discrepancy between the different selves can cause distress. Thus, black women who perceive themselves as underweight from the ideal body image standard of their ethnic group may display similar bingeing characteristics as the white women who perceive themselves as overweight relative to their ideal body image standards. Just the same, black women who are overweight from their ideal body image might engage in similar bingeing and purging characteristics as white women. Thus, these black women may show symptoms of disordered eating, body dissatisfaction, and negative emotions like white women.

A few studies have investigated this notion and found that black women are just as likely to engage in binge eating as white women, without displaying other eating disorder symptoms (Pinkowish, 1995) and engage in more weight gain than weight loss efforts (Rosen \& Gross, 1987). Thus, although black women are less likely to have eating disorders compared with white women, this does not mean that they are protected from eating disorder symptoms or ideal body image pressures.

In this study, we investigated the differing roles of body image perception, ideal body image perception, body image dissatisfaction, and bulimic symptoms among white and black women. Consistent with previous research, we hypothesized that (1) black women would endorse larger body images for their ideal body image perception of their ethnic group and their self-perceived body size compared with white women; (2) in terms of type of dissatisfaction, we hypothesize that white women will tend to report being overweight relative to their ideal body image, whereas black women will tend to report being underweight relative to their ideal. Regardless of type of body dissatisfaction, we also hypothesize that white women in general will tend to report more body image dissatisfaction compared with black women. Our main interest is to investigate how body image dissatisfaction relates to bulimic symptoms in both ethnic groups; (3) we hypothesize that black women who perceive themselves as larger or smaller than the ideal body size for their ethnic group will endorse bulimic symptoms compared with those who perceive their body size near the ideal body size for their ethnic group. For white women we hypothesize that those who consider themselves overweight relative to their ideal standards will report bulimic symptoms. Given that the ideal body image for white women is thin, we do not expect to find a significant amount of white women who consider themselves underweight from the ideal standard and engaging in bingeing behaviors. 
Overall, we predicted a curvilinear relationship between body image dissatisfaction and bulimic symptoms among black women and a linear relationship between body image dissatisfaction and bulimic symptoms among white women.

\section{METHOD}

\section{Participants}

Participants were 96 female undergraduate university students, who participated in the study to partially fulfill a requirement for their introductory psychology class. The ethnic composition of the sample was $62 \%$ white $(n=60)$, and $38 \%$ black $(n=36)$.

\section{Procedure}

Participants were informed that they would be filling out questionnaires about their emotions and self-perceptions. All participants completed written informed consents. Administration was conducted in groups of approximately 20. On completion of questionnaires, participants were debriefed by means of a written information sheet.

\section{Materials}

\section{Body Image Scale (Stunkard, Sorenson, \& Schulsinger, 1983)}

Seven female adult figure drawings, obtained from the Stunkard Body Figure Scale, were used to illustrate body weight ranging from very thin to obese. Participants indicated where on the scale they perceived their own body image and the ideal body image of women in their ethnic group. Reliability for these scales was reported as 0.74 (Sorenson et al., 1983; Stunkard et al., 1983). For validity coefficients and information see Sorenson et al. (1983).

To test how the participants viewed their own body image versus their perception of the ideal body image of their ethnic group, we computed difference scores. The difference score, BI/Self-Ethnic, is the participant's self-perception of their own body image minus her perception of the ideal body image of her ethnic group, where positive scores indicate the self-perception of being overweight and negative scores indicate the selfperception of being underweight.

\section{Eating Disorder Inventory (EDI; Garner, Olmsted, \& Polivy, 1983)}

The Eating Disorders Inventory is a 64-item, self-report measure of eating behavior and thought patterns. It has eight subscales: drive for thinness, bulimia, body dissatisfaction, ineffectiveness, perfectionism, interpersonal distrust, interoceptive awareness, and maturity fears. The subscales have shown adequate internal consistency coefficients and have been well validated. This study mainly focused on the bulimia subscale, which consists of six binge items and one purge item. Because the scale mostly assesses bingeing, we deleted the one purge item from the subscale (but results using the full scale were similar). The bulimia subscale without the purge item has an alpha coefficient of 0.82 .

\section{RESULTS}

Zero-order correlations and means and standard deviations of all variables for the entire sample are summarized in Table 1. As Table 1 shows, self-perceived body image 
Table 1. Intercorrelations, means, and standard deviations for the entire sample

\begin{tabular}{lccccr}
\hline \multicolumn{1}{c}{ Variables } & 1 & 2 & 3 & 4 & 5 \\
\hline 1. BI/Self & 1.00 & & & \\
& 3.68 & & & \\
2. BI/Ethnic & 0.91 & 1.00 & & \\
& 0.08 & 3.46 & & \\
3. BI/Self-Ethnic & & 0.66 & 1.00 & 1.00 & \\
& $0.80^{\mathrm{a}}$ & $-0.54^{\mathrm{a}}$ & 1.08 & & \\
4. Bulimia & & & & & \\
& $0.23^{\mathrm{b}}$ & $-0.35^{\mathrm{a}}$ & & & \\
& & & & & \\
5. Race & 0.00 & $0.66^{\mathrm{a}}$ & $-0.40^{\mathrm{a}}$ & $-0.35^{\mathrm{a}}$ & 1.00 \\
\hline
\end{tabular}

Note: $\mathrm{BI} /$ Self = self-perceived body image of participant; BI/Ethnic = ideal body image of participant's ethnic group; BI/Self-Ethnic = difference score between BI/Self and BI/Ethnic; Bulimia = bulimia subscale from Eating Disorder Inventory.

${ }_{\mathrm{a}}^{\mathrm{a}}<0.01$
$\mathrm{~b}_{p}<0.05$

(BI/Self) correlated with the bulimia subscale, such that the larger the body image size, the more bulimic symptoms were reported. BI/Ethnic also correlated with the bulimia subscale, such that the smaller the race-based ideal body image endorsed, the more bulimic symptoms were reported. BI/Self-Ethnic correlated with bulimic symptoms, where the larger the BI/Self-Ethnic score the more bulimic symptoms were endorsed. Thus, women who perceived themselves as overweight (positive scores) endorsed more bulimic symptoms than those who reported being underweight (negative scores). This finding will be further discussed in a later section. Race did not correlate with BI/Self, indicating that the groups did not differ on self-perceived body image. Race was correlated with BI/Ethnic, such that the black women reported larger ideal body images for their ethnic group. Race also correlated with BI/Self-Ethnic, such that white women tended to report larger differences between their body image and their ideal body image. The white women tended to report being overweight more so than black women. Finally, race correlated with the bulimia subscale, such that white women tended to report more bulimic symptoms than black women.

Zero-order correlations and means and standard deviations of all variables for each ethnic group are summarized in Table 2. As Table 2 shows, among the white group, BI/ Self was correlated with BI/Self-Ethnic such that the larger the self-perceived body size, the larger the perceived discrepancy between their body size and their ideal body size. In addition, BI/Ethnic correlated with BI/Self-Ethnic such that the smaller the ideal body image for their ethnic group, the larger the perceived discrepancy between the women's body image and their ideal body image. Among the white group, BI/Self-Ethnic correlated with bulimic symptoms in the predicted direction, where the larger the discrepancy score (meaning self-perception of more overweight relative to ethnic ideal) the more bulimic symptoms were endorsed, but the correlation was not significant (also, as will be shown later, the relation between BI/Self-Ethnic and bulimic symptoms among white women was curvilinear in form).

Among the black women, BI/Self correlated with BI/Self-Ethnic, such that the larger the self-perceived body size, the larger the discrepancy between their body size and their 
Table 2. Intercorrelations, means, and standard deviations for each ethnic group

\begin{tabular}{|c|c|c|c|c|}
\hline Variables & 1 & 2 & 3 & 4 \\
\hline \multicolumn{5}{|l|}{ White group } \\
\hline \multirow[t]{3}{*}{ 1. BI/Self } & 1.00 & & & \\
\hline & 3.68 & & & \\
\hline & 0.79 & & & \\
\hline \multirow{3}{*}{ 2. BI/Ethnic } & 0.11 & 1.00 & & \\
\hline & & 3.12 & & \\
\hline & & 0.52 & & \\
\hline \multirow[t]{3}{*}{ 3. BI/Self-Ethnic } & $0.82^{\mathrm{a}}$ & $-0.49^{\mathrm{a}}$ & 1.00 & \\
\hline & & & 0.55 & \\
\hline & & & 0.90 & \\
\hline \multirow{3}{*}{ 4. Bulimia } & 0.03 & -0.25 & 0.18 & 1.00 \\
\hline & & & & 12.25 \\
\hline \multirow{2}{*}{\multicolumn{5}{|c|}{ Black group }} \\
\hline & & & & \\
\hline \multirow{3}{*}{ 1. BI/Self } & 1.00 & & & \\
\hline & 3.68 & & & \\
\hline & 1.09 & & & \\
\hline \multirow[t]{3}{*}{ 2. BI/Ethnic } & 0.10 & 1.00 & & \\
\hline & & 4.02 & & \\
\hline & & 0.45 & & \\
\hline \multirow[t]{3}{*}{ 3. BI/Self-Ethnic } & $0.92^{\mathrm{a}}$ & -0.31 & 1.00 & \\
\hline & & & -0.33 & \\
\hline & & & 1.14 & \\
\hline \multirow[t]{2}{*}{ 4. Bulimia } & $0.53^{\mathrm{a}}$ & -0.01 & $0.51^{\mathrm{a}}$ & 1.00 \\
\hline & & & & $\begin{array}{l}9.08 \\
4.09\end{array}$ \\
\hline
\end{tabular}

ideal body size. BI/Self-Ethnic was also correlated with bulimia among the black women, such that the larger the discrepancy between their body size and their ideal body size, the more bulimic symptoms that were endorsed. Similar to the white group, positive BI/SelfEthnic scores represent overweight self-perception and negative BI/Self-Ethnic scores represent underweight self-perceptions. Thus, black women who perceived themselves as overweight endorsed more bulimic symptoms than those who perceived themselves as underweight. In addition, the black women's self-perceived body size (BI/Self) correlated with bulimic symptoms such that the larger the self-perceived body size, the more bulimic symptoms that were endorsed (but here again, as we report later, the relation between BI/ Self-Ethnic and bulimic symptoms among black women was curvilinear in form).

\section{Self-Perceived Body Image and Ideal Body Image Across Ethnic Groups}

The correlation between the participant's self body image perception (BI/Self) and their race (race $r=0.00, p=$ n.s.) reveals no systematic relation between race and body size. In line with prediction, the groups differed on the ideal body image of their ethnic group (BI/Ethnic; $r=0.66, p<0.01$ ), such that black women reported larger ideal body image sizes for their ethnic group $(M=4.02)$ than white women for their ethnic group $(M=3.12)$. 


\section{Body Image Dissatisfaction Across Ethnic Groups}

To determine the effect of race on participants' views of themselves versus the ideal body image of their ethnic group, we conducted a one-way analysis of variance (ANOVA). The ANOVA produced a significant effect for race, $F(1,92)=17.06, p<0.01$. Not surprisingly, the white female group reported the most overweight from the ideal body image of their ethnic group $(M=0.55)$, and the black group reported being underweight from the ideal body image of their ethnic group $(M=-0.33){ }^{1}$

\section{Body Image Dissatisfaction as a Predictor of Bulimic Symptoms Within Each Ethnic Group}

To evaluate our predictions on body image dissatisfaction and bulimic symptoms in black and white women, we constructed one block-wise hierarchal regression equation (see Table 3). In the first block, the equation consisted of the participant's race, selfperception of their body image minus their perception of the ideal body image of their ethnic group (BI/Self-Ethnic), and the squared term of BI/Self-Ethnic, to test for curvilinear relationships in predicting bulimic symptoms. The second block consisted of the two-way interactions between race and body image dissatisfaction (BI/Self-Ethnic) and the interaction between race and squared term.

The first block was significant overall $F(3,90)=11.96, p<0.01$. As Table 3 shows, all three predictors, race, body image dissatisfaction (BI/Self-Ethnic), and the squared term, were significant (Race partial correlation $=-0.29, t=-2.85, p<0.01$; BI/Self-Ethnic partial correlation $=0.24, t=2.31, p<0.05$; squared term partial correlation $=0.31, t=3.10$, $p<0.01-$ a positive $t$ value suggests the curvilinear shape to be in a U-shaped form). The effect for race reflected the fact that white women had a higher mean (12.25) than the

Table 3. Blockwise regression in predicting bulimic symptoms

\begin{tabular}{|c|c|c|c|c|c|}
\hline $\begin{array}{l}\text { Order } \\
\text { of Entry } \\
\text { of Set }\end{array}$ & $\begin{array}{l}\text { Predictors } \\
\text { in Set }\end{array}$ & $F$ for Set & $\begin{array}{l}t \text { for Within } \\
\text { Set Predictors }\end{array}$ & $d f$ & $\begin{array}{l}\text { Partial Correlation } \\
\text { (PR/pr) }\end{array}$ \\
\hline \multirow[t]{4}{*}{1.} & Main effects & $11.96^{\mathrm{a}}$ & & 3,90 & 0.29 \\
\hline & Race & & $-2.85^{\mathrm{a}}$ & 90 & -0.29 \\
\hline & BI/Self-Ethnic & & $2.31^{b}$ & 90 & 0.24 \\
\hline & Squared term & & $3.10^{\mathrm{a}}$ & 90 & 0.31 \\
\hline \multirow[t]{3}{*}{2.} & Two-way Interactions & 2.94 & & 2,88 & \\
\hline & Race $\times$ BI/Self-Ethnic & & 1.97 & 88 & 0.21 \\
\hline & Race $\times$ squared term & & 0.63 & 88 & 0.07 \\
\hline
\end{tabular}

Note: $N=94 ; \mathrm{PR}=$ partial correlation for set of predictors; $\mathrm{pr}=$ partial correlation for within set predictors; $\mathrm{BI} /$ Self-Ethnic = body image dissatisfaction term; Squared term = BI/Self-Ethnic squared; constructed to test for curvilinear relationship; Race $\times$ BI/Self-Ethnic $=$ interaction term between race and body image dissatisfaction; Race $\times$ squared term $=$ interaction term to test for ethnic group differences in curvilinear relationship.

${ }^{a} p<0.01$.

${ }^{\mathrm{b}} p<0.05$.

\footnotetext{
${ }^{1}$ The correlational approach was not used for this analysis, because we not only wanted to capture the magnitude and direction of the relationship between race and BI/Self-Ethnic but the large discrepancy between the means of each ethnic group on the BI/Self-Ethnic variable.
} 
black women (9.08) on the bulimic symptoms measure. In accord with predictions, body image dissatisfaction predicted bulimic symptoms. In addition, the relationship between body image dissatisfaction and bulimia is such that women who endorse being underweight or overweight relative to their ethnic body image idea, report more bulimic symptoms than women who do not endorse body image dissatisfaction, as reflected by the significant and positive curvilinear effect.

The second block with the interaction terms was not significant overall, $F(2,88)=2.94$, $p=$ n.s. The interaction between race and body image dissatisfaction was not a significant predictor of bulimic symptoms ${ }^{2}$ nor was the interaction between race and the squared term. We expected to find a curvilinear relationship among the black women; however, our data showed that this curvilinear relationship exists among both black and white women.

\section{DISCUSSION}

To our knowledge, there have been no previous studies to investigate curvilinear relationships between body image dissatisfaction and bulimic symptoms within black and white women. Thus, the primary contribution of our study involved our findings regarding the relationship between body image dissatisfaction and bulimic symptoms among different races. In this sample, ideal body image differed among white and black women, with the white women choosing thinner ideal body images. When comparing the participant's body size with their ideals, the white women tended to report being overweight, whereas the black women tended to report being underweight. In terms of bulimic symptoms, race was a significant predictor, such that white women reported significantly more symptoms than black women. Body image dissatisfaction was also a significant predictor of bulimia, such that the more overweight the women perceived themselves, the more bulimic symptoms that were endorsed. Interestingly, the curvilinear relationship between body image dissatisfaction and bulimia also emerged as a significant predictor of bulimic symptoms. This curvilinear relationship did not differ between the races. Women who considered themselves underweight or overweight relative to ethnic ideals endorsed more bulimic symptoms than those who reported little to no body image dissatisfaction.

Before discussing some implications of these findings, we first discuss some considerations and limitations in regard to this study. First, our measures were based on self-report, and no actual measure of body mass index was assessed. Thus, our study pertains solely to self-perceptions, and we have no measure to assess how accurate the self-perceptions are. Second, our sample consisted of undergraduates, limiting the generalizability of this study to nonclinical samples; on the other hand, bulimic symptoms and diagnoses are relatively common among undergraduate women. Third, no other variables other than body image self-perceptions, body image dissatisfaction, and bulimic symptoms were obtained. It is possible that white and black women differ on other variables (e.g., self-esteem, self-efficacy, perfectionism) that contribute to bulimic symptoms. This necessitates further research.

\footnotetext{
${ }^{2}$ This interaction approached significance $(p=0.052)$ and reflects the ethnic difference between the correlations of BI/Self-Ethnic and bulimic symptoms (Table 2, white women, $r=0.18$; black women, $r=0.51$ ). Nonetheless, this interaction should be interpreted in the context of the curvilinear findings.
} 
Our findings may have some conceptual and clinical implications. Past research has suggested that black women may be buffered against eating disorder symptoms caused, in part, by the loyalty to their culture. Although eating disorders are not especially prevalent among black women, our findings suggest that if black women are dissatisfied with their body image (either viewing themselves as underweight or overweight), then they are not buffered against bulimic symptoms. Black women may pressure themselves to achieve their cultural standards of ideal body size just like white women.

Past research has focused on women who consider themselves overweight. Our findings suggest that women who consider themselves underweight may engage in bingeing behaviors. The bingeing behavior might serve as a compensation for being smaller than ideal rather than the usual compensatory behavior of purging because of feeling overweight or overeating. Another possibility might be that because women perceive themselves as smaller than the ideal, this causes negative affect, and bingeing occurs as an attempt to escape the negative affect (Heatherton \& Baumeister, 1991). Differentiating what mechanisms are at work for women who binge but consider themselves underweight is an interesting area for future research.

Our findings provide further evidence for the relationship between body dissatisfaction and disordered eating. Most of the literature in this area has focused on linear relationships between body image dissatisfaction and bulimia, in which higher body image dissatisfaction predicts more bulimic symptoms. Our data suggest that this is not always the case, and failure to assess for curvilinear relationships between body image dissatisfaction and bulimia may possibly lead to misleading conclusions. For example, assessing only linear relationships between body image dissatisfaction and bulimic symptoms might lead one to conclude that only women who are overweight demonstrate bulimic symptoms, which, given our findings, is not always the case.

Finally, future research should investigate the role cultural standards play in body image and disordered eating in women. In particular, more research is needed on the group of individuals who are underweight and endorse bulimic symptoms. Is there a difference in eating disorder symptoms among women who are overweight and those who are underweight? As was suggested earlier, the difference may involve purging. Women who consider themselves overweight might engage in purging behaviors to compensate for their bingeing behaviors. However, women who consider themselves underweight may not exhibit purging behaviors, because this obstructs their goal of gaining weight.

In conclusion, this study predicted and found that ideal body image perceptions vary across ethnic groups. Despite this ethnic group difference, we found that both black and white women who are dissatisfied with their body image (underweight or overweight) may be predisposed to bulimic symptoms. These results may inform the understanding and assessment of disordered eating in young women.

\section{REFERENCES}

Abrams, K.K., Allen, L.R., \& Gray, J.J. (1993). Disordered eating attitudes and behaviors, psychological adjustment, and ethnic identity: A comparison of black and white female college students. International Journal of Eating Disorders, 14, 49-57.

Akan, G.E., \& Grilo, C.M. (1995). Sociocultural influences on eating attitudes and behavior, body image and psychological functioning: A comparison of African American, Asian American and Caucasian college women. International Journal of Eating Disorders, 18, 181-187.

Alan, J.D., Mayo, K., \& Michel, Y. (1993). Body size values of white and black women. Research in Nursing \& Health, 16, 323-333. 
Butler, J.C., \& Ryckman, R.M. (1993). Perceived and ideal physiques in male and female university students. The Journal of Social Psychology, 133, 751-752.

Casper, R.C., \& Offer, D. (1990). Weight and dieting concerns in adolescents: Fashion or symptom? Pediatrics, $86,384-390$.

Garner, D.M., Olmstead, M.P., \& Polivy, J. (1983). Development and validation of a multidimensional eating disorder inventory for anorexia nervosa and bulimia. International Journal of Eating Disorders, 2, $15-34$.

Heatherton, T.F., \& Baumeister, R.F. (1991). Binge eating as escape from self-awareness. Psychological Bulletin, $110,86-108$.

Higgins, E.T. (1987). Self-discrepancy: A theory relating self and affect. Psychological Review, 94, 319-340.

Lamb, C.S., Jackson, L.A., Cassiday, P.B., \& Priest, D.J. (1993). Body figure preferences of men and women: A comparison of two generations. Sex Roles, 28, 345-358.

Molloy, B.L., \& Herzberger, S.D. (1998). Body image and self-esteem: A comparison of African-American and Caucasian women. Sex Roles, 38, 631-643.

Myers, P.N., \& Biocca, F.A. (1992). The elastic body image: The effect of television advertising and programming on body image distortions in young women. Journal of communication, 42, 108-134.

Parker, S., Nichter, M., Nicther, M., \& Vuckovic, N. (1995). Body image and weight concerns among AfricanAmerican and Caucasian adolescent females: Differences that make a difference. Human Organization Sum, 54, 103-114.

Pinkowish, M. (1995). Eating disorders: No stereotypes need apply. Patient Care, 29, 13.

Rosen, J.C., \& Gross, J. (1987). Prevalence of weight reducing and weight gaining in adolescent girls and boys. Health Psychology, 6, 131-147.

Rucker, C.E., \& Cash, T.F. (1992). Body images, body-size perceptions and eating behaviors among AfricanAmerican and white college women. International Journal of Eating Disorders, 12, 291-299.

Sorenson, T.I.A., Stunkard, A.J., Teasdale, T.W., \& Higgins, M. (1982). The accuracy of reports of weight: Children's recall of their parents' weight 15 years earlier. International Journal of Obesity, 6, 79-84.

Stice, E., \& Shaw, H.E. (1994). Adverse effects of the media portrayed thin-ideal on women and linkages to bulimic symptomatology. Journal of Social and Clinical Psychology, 13, 288-308.

Stunkard, A.J., Sorenson, T., \& Schlusinger, F. (1983). Use of the Danish adoption register for the study of obesity and thinness. In S. Kety, L.P. Rowland, R.L. Sidman, \& S.W. Matthysse (Eds.), The genetics of neurological and psychiatric disorders. New York: Raven Press.

Usmiani, S. \& Daniluk, J. (1997). Mothers and their adolescent daughters: Relationship between self-esteem, gender role identity, and body image. Journal of Youth and Adolescence, 26, 45-62. 